\title{
Inter-network connectivity and amyloid- beta linked to cognitive decline in preclinical Alzheimer's disease: a longitudinal cohort study
}

Roy W. E. Van Hooren ${ }^{1^{*}}$ D, Joost M. Riphagen ${ }^{1,2}$, Heidi I. L. Jacobs ${ }^{1,3,4}$ and For the Alzheimer's Disease Neuroimaging Initiative

\begin{abstract}
Background: Amyloid-beta (A $)$ has a dose-response relationship with cognition in healthy adults. Additionally, the levels of functional connectivity within and between brain networks have been associated with cognitive performance in healthy adults. Aiming to explore potential synergistic effects, we investigated the relationship of inter-network functional connectivity, A $\beta$ burden, and memory decline among healthy individuals and individuals with preclinical, prodromal, or clinical Alzheimer's disease.

Methods: In this longitudinal cohort study (ADNI2), participants (55-88 years) were followed for a maximum of 5 years. We included cognitively healthy participants and patients with mild cognitive impairment (with or without elevated $A \beta$ ) or Alzheimer's disease. Associations between memory decline, $A \beta$ burden, and connectivity between networks across the groups were investigated using linear and curvilinear mixed-effects models.

Results: We found a synergistic relationships between inter-network functional connectivity and A $\beta$ burden on memory decline. Dose-response relationships between $A \beta$ and memory decline varied as a function of directionality of internetwork connectivity across groups. When inter-network correlations were negative, the curvilinear mixed-effects models revealed that higher $A \beta$ burden was associated with greater memory decline in cognitively normal participants, but when inter-network correlations were positive, there was no association between the magnitude of $A \beta$ burden and memory decline. Opposite patterns were observed in patients with mild cognitive impairment. Combining negative inter-network correlations with $A \beta$ burden can reduce the required sample size by $88 \%$ for clinical trials aiming to slow down memory decline.
\end{abstract}

Conclusions: The direction of inter-network connectivity provides additional information about $A \beta$ burden on the rate of expected memory decline, especially in the preclinical phase. These results may be valuable for optimizing patient selection and decreasing study times to assess efficacy in clinical trials.

Keywords: Alzheimer's disease, Amyloid- $\beta$, Cognitively normal, Inter-network functional connectivity, Longitudinal, Memory performance, Mild cognitive impairment, Preclinical, Prodromal, Clinical trials

\footnotetext{
* Correspondence: roy.vanhooren@maastrichtuniversity.nl

'Faculty of Health, Medicine and Life Sciences; School for Mental Health and

Neuroscience, Department of Psychiatry and Neuropsychology, Alzheimer

Center Limburg, Maastricht University, Dr. Tanslaan 12, 6229 ET Maastricht,

the Netherlands

Full list of author information is available at the end of the article
}

(c) The Author(s). 2018 Open Access This article is distributed under the terms of the Creative Commons Attribution 4.0 International License (http://creativecommons.org/licenses/by/4.0/), which permits unrestricted use, distribution, and reproduction in any medium, provided you give appropriate credit to the original author(s) and the source, provide a link to the Creative Commons license, and indicate if changes were made. The Creative Commons Public Domain Dedication waiver (http://creativecommons.org/publicdomain/zero/1.0/) applies to the data made available in this article, unless otherwise stated. 


\section{Background}

Elevated levels of amyloid-beta (A $\beta)$, a neuropathological hallmark of Alzheimer's disease (AD) [1-6], are crucial in identifying the earliest stages of $\mathrm{AD}$. While $\mathrm{A} \beta$ does not relate well to cognition cross-sectionally, it has a dose-response relationship with cognition in healthy adults [7]. However, dementia-related pathologies, including $A \beta$, only explain $41 \%$ of variation in cognitive decline [8] and a low signal-to-noise ratio of biomarkers in the asymptomatic stages of the disease for entry criteria in clinical trials has been reported as a reason for trial failure [9].

Healthy aging has previously been associated with reduced activity within the default mode network (DMN), a network often associated with memory-related processes such as thinking about the future, episodic memory, and autobiographical memory [10]. Reduced connectivity within the DMN, as measured with resting-state functional magnetic resonance imaging, is also associated with impaired cognitive performance in old age [11]. Additionally, levels of functional connectivity within cognition-related intrinsic brain networks, such as the $\mathrm{DMN}$, and $\mathrm{A} \beta$ burden have a synergistic effect on memory decline in clinically normal older individuals [12]. Various networks predict cognitive decline which may indicate that $A \beta$ burden impacts the interaction between networks. Negative correlations between the DMN and task-positive networks has been positively associated with cognitive performance in young individuals $[13,14]$. These negative correlation patterns are also referred to as "anti-correlations" in the literature and have been described as intrinsically organized antagonistic activation patterns between networks in the brain [15]. These patterns are reported to be part of a mechanism that facilitates cognition, possibly by reinforcing connections between two loci in the brain dedicated to cognitive functions $[14,16]$.

Previous studies have reported attenuated effects of aging with negative correlations between resting-state networks, with negative correlations further decreasing in patients with mild cognitive impairment (MCI) and $\mathrm{AD}$ [17-19]. While detrimental effects of $\mathrm{A} \beta$ on cognition and on functional connectivity within networks have been shown [2, 20-22], it remains unknown whether $A \beta$ modulates functional connectivity between networks. Given the close relationship between inter-network connectivity and cognitive decline and these first reported associations with $\mathrm{A} \beta$, combining information from inter-network connectivity with $A \beta$ may reduce noise when selecting asymptomatic individuals at risk for $\mathrm{AD}$ in clinical trials aimed at slowing down cognitive decline.

To that end, we investigated whether functional connectivity between the DMN and task-positive networks predicts memory decline differently among cognitively normal individuals, MCI patients with and without elevated $\mathrm{A} \beta$, or $\mathrm{AD}$ patients. Additionally, we investigated whether the relationship between inter-network connectivity and memory decline depends on $A \beta$ levels in a dose-response type relationship. We expected that, as the magnitude of negative correlations decreases, memory performance would be lower, and that the strength of these associations would show a dose-response relationship with $A \beta$ burden. To investigate domain-specificity of our findings, we have also investigated our hypotheses using executive functions as a control outcome measure.

\section{Methods}

Data used in this article were obtained from the Alzheimer's Disease Neuroimaging Initiative (ADNI) database (adni.loni.usc.edu). The ADNI was launched in 2003, led by Principal Investigator Michael W. Weiner, MD. The main goal of the ADNI is to test whether magnetic resonance imaging, positron emission tomography, other biological markers, and clinical and neuropsychological assessment can be combined to measure the progression of MCI and AD. For up-to-date information, see www.adni-info.org.

\section{Participants}

We included data from a total of 122 eligible participants from the ADNI2 study (as of March 2017), of which seven were removed due to low imaging data quality, resulting in a total of 115 participants. Qualified clinicians working for the ADNI categorized participants into four groups (cognitively normal, early $\mathrm{MCI}$, late $\mathrm{MCI}$, or $\mathrm{AD}$ ) based on diagnostic procedures from the ADNI protocol [23]. However, for the purposes of our study, we grouped all patients with MCI based on their $\mathrm{A} \beta$ levels being below or above a pathological cutoff point of $1.11{ }^{18} \mathrm{~F}-\mathrm{AV}-45$ florbetapir positron emission tomography standardized uptake value ratio [24], respectively (referred to as $\mathrm{MCI}^{-}$and $\mathrm{MCI}^{+}$in this manuscript). MCI diagnoses were based on the Petersen criteria for MCI $[25,26]$, while patients with AD met the NINCDS-ADRDA criteria for probable Alzheimer's disease [27]. For a detailed overview of exclusion, inclusion, and diagnostic criteria and procedures, please refer to the ADNI2 protocol (http://adni.loni.usc.edu/wp-content/uploads/2008/07/adni2-procedures-manual.pdf). Additionally, only participants with complete resting-state functional magnetic resonance imaging and $A \beta$ data at baseline were eligible for inclusion, and patients with $\mathrm{AD}$ were only eligible if their $A \beta$ levels were above the cutoff point to ensure only AD-related pathology was represented in this group. A complete listing of all participant IDs that were included in the final analyses can be found in Additional file 1 (Table S1). 


\section{Materials and equipment Test batteries}

The main outcome measures we used were the composite memory score (ADNI-Mem) and the composite executive functions score (ADNI-EF), which have good validity and are ideally suited to track changes over time. These scores were derived by combining scores related to memory performance and executive functions. ADNI-Mem score is derived from several test batteries, including the Rey Auditory Verbal Learning Test, Alzheimer's Disease Assessment Schedule-Cognition, Mini-Mental State Examination, and Wechsler Memory Scale-Revised [28]. ADNI-EF score is derived from tests including WAIS-R Digit Symbol Substitution, Digit Span Backwards, Trails A and B, Category Fluency, and Clock Drawing [29].

\section{Biomarker assessment}

${ }^{18} \mathrm{~F}-\mathrm{AV}-45$ florbetapir positron emission tomography measures were used to quantify levels of neocortical $A \beta$ at baseline. The duration of positron emission tomography imaging was $20 \mathrm{~min}$ and started $50 \mathrm{~min}$ after injection of tracer fluid. The neocortical standardized uptake value ratio is the mean uptake in an aggregate of the frontal lobe, cingulate cortex, lateral parietal, and lateral temporal regions relative to mean uptake in the whole cerebellum, including white and gray matter. Further processing of positron emission tomography images was performed as described in a previous report [24]. Participants were characterized as $\mathrm{A} \beta$-positive if they exceeded the cutoff value of 1.11 standardized uptake value ratio, as previously determined in ADNI cohorts [24].

\section{Imaging equipment and acquisition}

Imaging data were acquired using Philips Medical Systems 3.0-Tesla magnetic resonance systems. Structural T1-weighted gradient echo pulse sequence data with dimensions of $170 \times 256 \times 256 \mathrm{~mm}$ with a voxel resolution of 1.2 $\times 1 \times 1 \mathrm{~mm}$ were acquired in sagittal orientation with a repetition time of $6.8 \mathrm{~ms}$, echo time of $3.1 \mathrm{~ms}$, flip angle of $9^{\circ}$, and slice thickness of $1.2 \mathrm{~mm}$. Additionally, resting-state functional magnetic resonance imaging scans of $7 \mathrm{~min}$ were obtained, consisting of 140 volumes of $\mathrm{T} 2 \%$-weighted data with 48 slices per volume, dimensions of $64 \times 64 \times 48 \mathrm{~mm}$, and a voxel resolution of $3.3 \times 3.3 \times 3.3 \mathrm{~mm}$. Functional images were acquired in transverse orientation with repetition time of $3000 \mathrm{~ms}$, echo time of $30 \mathrm{~ms}$, flip angle of $80^{\circ}$, and a slice thickness of $3.3 \mathrm{~mm}$.

\section{Image preprocessing and de-noising}

For preprocessing we used the default preprocessing pipeline for volume-based analyses within the NITRC CONN toolbox (version 17.a; http://www.nitrc.org/projects/conn/) [30]. Images were realigned and unwarped, centered, slice-time corrected, segmented (gray/white/cerebrospinal fluid), normalized to MNI space, and outliers were identified with the Artifact Detection Toolbox. Outliers were regressed out (scrubbing) using conservative settings (95th percentile in normative sample, global signal $\mathrm{Z}$ value $=2$, motion $=0.5 \mathrm{~mm}$, and we discarded the first three volumes). Functional data were smoothed with a Gaussian kernel of $6 \mathrm{~mm}$ full width at half maximum. As the reliability and validity of negative correlation measures has been the subject of debate [31], de-noising of data involved regressing out principal components of the signal from white matter and cerebrospinal fluid following the CompCor method [32] which reduces the presence of artificial negative correlations. Additionally, linear detrending and an after-regression BOLD signal band-pass filter $(0.008<\mathrm{f}$ $<0.09 \mathrm{~Hz}$ ) were applied. Data quality was ensured by visual inspection of histograms of functional connectivity values before and after de-noising for each participant. Histograms that did not show a normal distribution of functional connectivity values after de-noising were indicative of a suboptimal de-noising process and these participants were excluded from data analysis to preserve a high level of data quality $(n=7)$. Motion parameters were entered as regressor in our analyses. Volumes with motion above $0.5 \mathrm{~mm}$ were entered as regressors in our general linear models ("scrubbing"). Furthermore, all images were inspected for irregularities during the entire preprocessing and analysis process.

\section{Functional connectivity analyses}

A bivariate correlation, hemodynamic response function-weighted ROI-to-ROI analysis was performed within CONN, using four independent network regions of interest (ROI) as sources, with a total of 19 structurally defined hub regions. The regions of interest include the DMN and regions representing task-positive networks, including the dorsal attention network (DAN), salience network (SN), and frontoparietal network (FPN). These regions of interest, which are part of the CONN software package, were generated by an independent component analysis on 497 healthy control participants (293 females) as part of the Human Connectome Project (http://www.humanconnectome.org) [30]. As a reference for future replication efforts, peak coordinates of the regions of interest used for this functional connectivity analysis are provided in Additional file 1 (Table S2) and the networks used are visualized in Fig. 1. To assess inter-network connectivity, the average time course signal from each network was extracted and Fisher r-to-z transformed inter-network correlation values were exported for statistical analyses.

\section{Statistical analysis}

All data were analyzed using $\mathrm{R}$ version 3.3.1 (https:// www.r-project.org/) and MATLAB R2016a. Baseline participant characteristics were compared with analysis of 


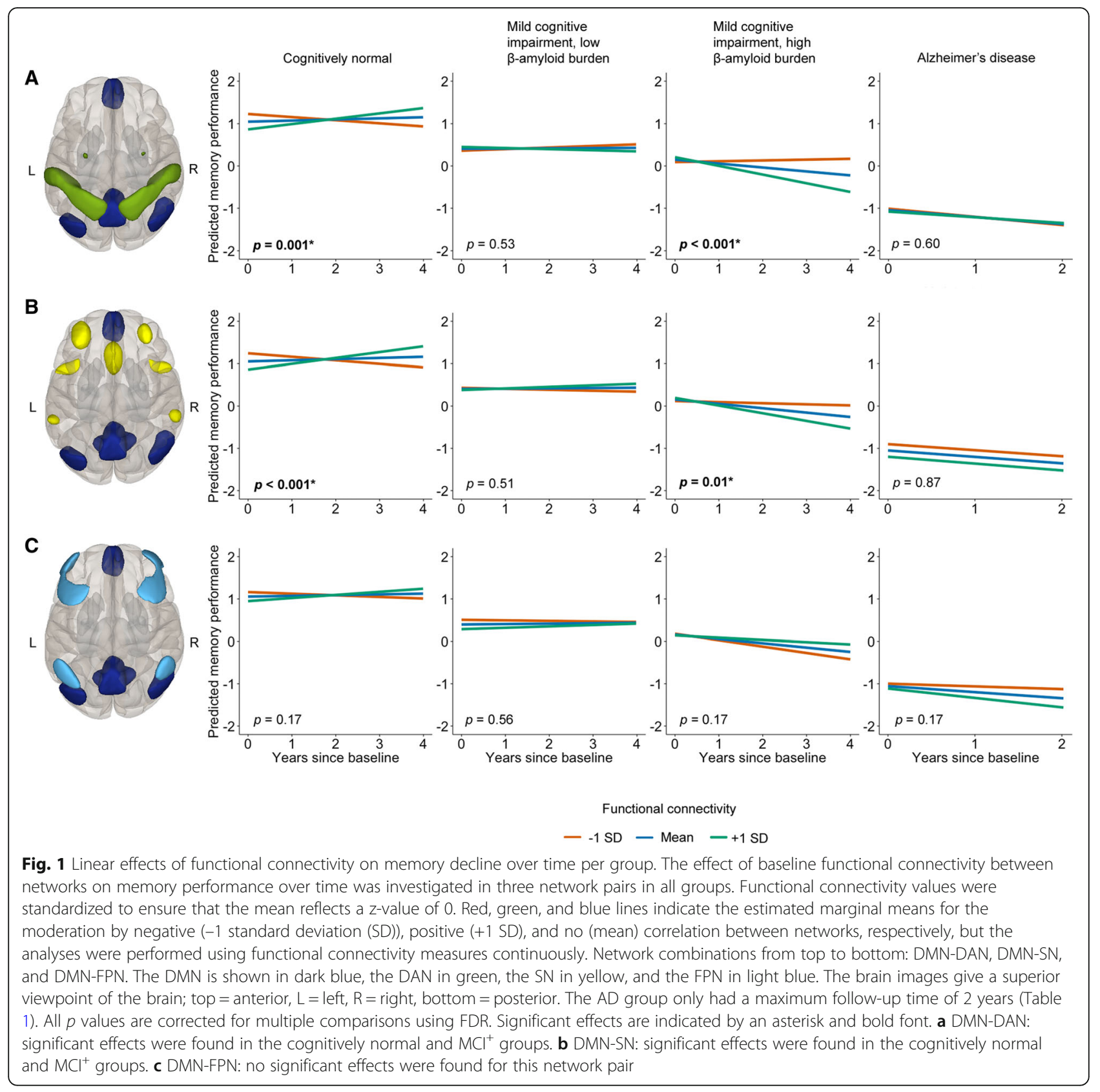

variance for continuous variables and chi-squared test for categorical variables. Linear regression analyses were used for cross-sectional data. Because of their ability to deal with missing data in longitudinal studies without being subject to complete-case bias [33, 34], longitudinal analyses were performed with linear and curvilinear mixed-effect regression analyses using maximum likelihood, utilizing nlme version 3.1-128 [35]. Fixed effects were predictor of interest, a random intercept for each participant, and random slope for time. Time was calculated as the number of years since baseline assessment.
During the construction of statistical models, we first investigated effects in the whole sample. In case of a significant effect, the sample was split based on clinical groups and the level of amyloid burden, for which interactive analyses were performed with the cognitively normal group as reference. We also performed within-group analyses to better understand the patterns. For all models, we compared the Akaike Information Criteria between models with a random intercept and random slope or a random intercept only using the Log-likelihood ratio test and selected the most parsimonious model. In all models, 
age, sex, education, and their interaction with time were included as covariates if $p<0.10$ (using the Wald $t$ statistic) [36]. To improve longitudinal data quality [37], variability in head size and intracranial volume, as measured with FreeSurfer version 5.1 [38], was added as a covariate [39]. Additionally, intracranial volume can be used as a measure of head size, which correlates with total gray matter atrophy. It is important to control for atrophy as atrophy can reduce blood flow [40], and $\mathrm{AD}$ patients may show more atrophy than patients with $\mathrm{MCI}$ or healthy controls. The most complex model constructed for analyses is described in detail in Additional file 1 (Box 1). Residual plots and Q-Q plots were examined for all models. Significance was set at $p<0.050$ (two-sided) and results were corrected for multiple comparisons using the false-discovery rate (FDR). Power calculations were performed using MATLAB scripts for the mixed-effects model using the slope of memory decline and residual variance [40] to estimate the number of participants a clinical trial would need to enroll to detect slowing of memory decline of $30 \%$ (two arms for 4-year annual assessments, $80 \%$ power, alpha $=0.05$ ) using high amyloid levels as an inclusion criterion. These mixed-effects models included the covariates, random intercept, and random slope.

\section{Results}

\section{Demographics}

The total sample consisted of 115 participants, including 53 females (46\%) with a mean age of 72.91 (standard deviation $(\mathrm{SD})=6.68)$ at baseline. Of these participants, 28 were in the cognitively normal group, 27 were in the $\mathrm{MCI}^{-}$group, 36 were in the $\mathrm{MCI}^{+}$group, and 24 were in the $\mathrm{AD}$ group. Other participant characteristics are summarized in Table 1.

Analyses of variance indicated group differences in ${ }^{18} \mathrm{~F}-\mathrm{AV}-45$ florbetapir standardized uptake value ratio, ADNI-Mem, ADNI-EF, clinical dementia rating scale scores, and follow-up times. The proportion of cases at each time point were as follows: baseline, 115 (100\%); month 6, 110 (96\%); month 12, 98 (85\%); month 24, 74 (64\%); month 36, 6 (5\%); month 48, 29 (25\%); and month $60,1(<1 \%)$. Results of Tukey tests for post-hoc differences between group characteristics are available in Additional file 1 (Table S3). Additionally, we found no differences in image preprocessing parameters between groups (Additional file 1: Table S4).

\section{Effects of functional connectivity and group on memory at baseline}

There were no significant associations between functional connectivity between networks and memory performance at baseline across the entire sample or within the groups. An association was found between functional connectivity between the DMN and FPN and executive functions across the entire sample at baseline. Further analyses of the association between functional connectivity and executive functions within the diagnosis groups reveal an association in the amyloid-negative MCI group (Additional file 1: Table S5).

Table 1 Summary table of participant characteristics at baseline

\begin{tabular}{|c|c|c|c|c|c|}
\hline Variable & $\mathrm{CN}(n=28)$ & $\mathrm{MCl}^{-}(n=27)$ & $\mathrm{MCl}^{+}(n=36)$ & $\mathrm{AD}(n=24)$ & $p$ value \\
\hline AV45 SUVR & $1.16(0.21)$ & $1.01(0.05)$ & $1.39(0.17)$ & $1.50(0.15)$ & $<0.001$ \\
\hline Age (years) & $74(5.71)$ & $71.36(8.03)$ & $72.58(5.79)$ & $74(7.32)$ & 0.42 \\
\hline Education (years) & $16.43(1.93)$ & $16.48(2.68)$ & $15.86(2.46)$ & $15.46(2.50)$ & 0.36 \\
\hline Females & $15(54 \%)$ & $12(44 \%)$ & 14 (39\%) & $12(50 \%)$ & 0.67 \\
\hline ICV $\left(\mathrm{mm}^{3}\right)$ & $1,572,034(153,316.20)$ & $1,545,103(168,923.10)$ & $1,568,839(174,101.60)$ & $1,574,218(210,862)$ & 0.94 \\
\hline APOE $\varepsilon 4$ & $10(36 \%)$ & $4(15 \%)$ & $24(66 \%)$ & $20(83 \%)$ & $<0.001^{\mathrm{a}}$ \\
\hline ADNI-Mem & $0.89(0.52)$ & $0.37(0.49)$ & $0.15(0.60)$ & $-1.10(0.56)$ & $<0.001$ \\
\hline ADNI-EF & $0.80(0.56)$ & $0.25(0.80)$ & $0.34(0.86)$ & $-0.94(0.62)$ & $<0.001$ \\
\hline CDR-SB & $0.05(0.16)$ & $1.26(0.92)$ & $1.90(1.06)$ & $4.41(1.31)$ & $<0.001$ \\
\hline FC DMN-DAN & $-0.12(0.28)$ & $0.00(0.33)$ & $-0.03(0.24)$ & $-0.15(0.30)$ & 0.17 \\
\hline FC DMN-SN & $-0.15(0.29)$ & $-0.11(0.28)$ & $-0.08(0.28)$ & $-0.18(0.27)$ & 0.56 \\
\hline FC DMN-FPN & $0.31(0.23)$ & $0.20(0.30)$ & $0.26(0.23)$ & $0.17(0.29)$ & 0.24 \\
\hline Follow-up time (years) & $1.63(1.24)$ & $1.30(0.96)$ & $1.46(1.09)$ & $0.90(0.53)$ & 0.001 \\
\hline
\end{tabular}

Data are presented as the mean (standard deviation) for the continuous variables and as $n$ (\%) for the categorical variable

The APOE $\varepsilon 4$ row gives the number of participants in this group with one or more APOE $\varepsilon 4$ alleles

Statistical significance was tested with analysis of variance for continuous variables and chi-squared test for the categorical variables

$A P O E \& 4$ apolipoprotein $\varepsilon 4, A D$ Alzheimer's disease, ADNI-EF Alzheimer's Disease Neuroimaging Initiative executive functions score composite, $A D N I-M e m$

Alzheimer's Disease Neuroimaging Initiative memory score composite, AV45 ${ }^{18} \mathrm{~F}-\mathrm{AV}$-45 florbetapir, CDR-SB Clinical Dementia Rating scale-sum of boxes, CN

cognitively normal, DAN dorsal attention network, DMN default mode network, FC functional connectivity, FPN frontoparietal network, ICV intracranial volume, $M C I$ mild cognitive impairment, $S N$ salience network, SUVR standardized uptake value ratio

${ }^{\text {a }}$ All groups were significantly different from each other, except $\mathrm{CN}$ versus $\mathrm{MCl}^{-}$and $\mathrm{MCl}^{+}$versus $A D$ 
Longitudinal linear effects of functional connectivity and group on memory

Linear mixed-effect models were performed to investigate effects of functional connectivity and group on memory decline over time. The associations between functional connectivity between the networks and time for the whole sample were not significant (Additional file 1: Table S6). Using the cognitively normal group as a reference level, a significant three-way interaction effect of time, group, and functional connectivity was found in comparison with the $\mathrm{MCI}^{+}$group for the DMN-DAN and DMN-SN correlations, but not for the DMN-FPN correlations (Table 2). Adding inter-network functional connectivity to the models with the covariates contributed significantly to the explained variance of memory decline for the DMN-DAN $\left(R^{2}\right.$ difference $=0.03,95 \%$ confidence interval $(\mathrm{CI}) 0.01$ to $0.07 ; p=0.04)$, but not for the DMN-SN $\left(R^{2}\right.$ difference $=$ $0.001,95 \%$ CI -0.001 to $0.01 ; p=0.21$ ) or the DMN-FPN ( $R^{2}$ difference $<0.001,95 \%$ CI -0.001 to $0.01 ; p=0.48$ ).

Figure 1 and Table 2 show significant associations between DMN-DAN and DMN-SN functional connectivity and memory decline between the cognitively normal and $\mathrm{MCI}^{+}$groups. Post-hoc linear mixed models within each group confirmed that, for the cognitively normal group, positive correlations between these network pairs are positively associated with memory performance over time, whereas in the $\mathrm{MCI}^{+}$group positive correlations were negatively associated with memory performance (Additional file 1: Table S7). No significant associations were found for executive functions (Additional file 1: Table S8).

As the cognitively normal group consisted of 11 individuals with $A \beta$ levels above the threshold, we also examined associations between functional inter-network connectivity, $A \beta$ as a continuous variable, and memory decline in the cognitively normal group. These results showed that this interaction was significant for the DMN-DAN and the DMN-SN, but not for the DMN-FPN. This shows that $\mathrm{A} \beta$ moderates the association between functional connectivity and memory decline also in the cognitively normal group in such a way that negative correlations were associated with memory decline with elevated $A \beta$ (Table 3). Similar findings are observed when grouping cognitively normal individuals based on the $A \beta$ cutoff value (Additional file 1: Table S9).

\section{Longitudinal curvilinear effects of functional connectivity and amyloid-beta on memory}

Since our linear mixed model results showed opposite moderations by $A \beta$ on the association between functional connectivity and memory performance across the diagnostic groups, we examined curvilinear (quadratic) mixed-effect models for these network interactions using $A \beta$ as a continuous variable to investigate a possible dose-response relationship with memory decline. To ensure that individuals with the highest $A \beta$ levels would not drive associations, we excluded the $\mathrm{AD}$ group from these analyses. In the whole sample, excluding the $\mathrm{AD}$ group, a quadratic three-way interaction effect of functional connectivity, $A \beta$, and time was found for the DMN-DAN, but marginally not for the DMN-SN correlations (Table 4). To visualize this interaction, we plotted simple slopes for different values of ${ }^{18} \mathrm{~F}-\mathrm{AV}-45$ florbetapir standardized uptake value ratio in both groups, broken down by positive and negative inter-network correlations and holding all other fixed effects constant (Fig. 2). Values for $A \beta$ were chosen to reflect $A \beta$

Table 2 Associations between group, inter-network functional connectivity, and memory decline

\begin{tabular}{|c|c|c|c|c|c|c|c|}
\hline Fitted model & Est. & SE & $95 \% \mathrm{Cl}$ & DF & T value & $p$ value & $p$ value (FDR) \\
\hline \multicolumn{8}{|c|}{ DMN-DAN ( $n=115$, number of observations $=433$ ) } \\
\hline Time $\times \mathrm{MCl}^{-} \times \mathrm{DMN}-\mathrm{DAN}$ & -0.43 & 0.22 & -0.68 to -0.01 & 310 & -2.00 & 0.047 & 0.14 \\
\hline Time $\times \mathrm{MCl}^{+} \times$DMN-DAN & -0.79 & 0.23 & -1.25 to -0.34 & 310 & -3.43 & 0.001 & 0.001 \\
\hline Time $\times A D \times D M N-D A N$ & -0.24 & 0.30 & -0.84 to 0.36 & 310 & -0.79 & 0.43 & 0.43 \\
\hline \multicolumn{8}{|c|}{ DMN-SN ( $n=115$, number of observations $=433$ ) } \\
\hline Time $\times \mathrm{MCl}^{-} \times \mathrm{DMN}-\mathrm{SN}$ & -0.26 & 0.23 & -0.71 to 0.19 & 310 & -1.13 & 0.26 & 0.32 \\
\hline Time $\times \mathrm{MCl}^{+} \times \mathrm{DMN}-\mathrm{SN}$ & -0.55 & 0.20 & -0.93 to -0.16 & 310 & -2.81 & 0.01 & 0.01 \\
\hline Time $\times A D \times D M N-S N$ & -0.36 & 0.31 & -0.98 to 0.25 & 310 & -1.17 & 0.25 & 0.37 \\
\hline \multicolumn{8}{|c|}{ DMN-FPN ( $n=115$, number of observations $=433$ ) } \\
\hline Time $\times \mathrm{MCl}^{-} \times \mathrm{DMN}-\mathrm{FPN}$ & -0.27 & 0.27 & -0.81 to 0.26 & 310 & -1.00 & 0.32 & 0.32 \\
\hline Time $\times \mathrm{MCI}^{+} \times$DMN-FPN & -0.21 & 0.26 & -0.72 to 0.30 & 310 & -0.81 & 0.42 & 0.42 \\
\hline Time $\times$ AD $\times$ DMN-FPN & -0.45 & 0.33 & -1.09 to 0.19 & 310 & -1.38 & 0.17 & 0.37 \\
\hline
\end{tabular}

Results are acquired using the cognitively normal group as a reference group

Regression models are adjusted for age, intracranial volume, sex, and education

Beta coefficients are unstandardized

$A D$ Alzheimer's disease, $C l$ confidence interval, DAN dorsal attention network, $D F$ degrees of freedom, DMN default mode network, FDR false discovery rate, FPN frontoparietal network, $\mathrm{MCl}$ mild cognitive impairment, $S E$ standard error, $S N$ salience network 
Table 3 Three-way interaction of functional connectivity, amyloid-beta, and time in the cognitively normal group

\begin{tabular}{|c|c|c|c|c|c|c|c|}
\hline Fitted model & Est. & SE & $95 \% \mathrm{Cl}$ & DF & T value & $p$ value & $p$ value (FDR) \\
\hline \multicolumn{8}{|c|}{ DMN-DAN $(n=28$, number of observations $=118)$} \\
\hline DMN-DAN $\times$ A $\beta \times$ Time & 1.97 & 0.48 & 1.03 to 2.92 & 86 & 4.14 & $<0.001$ & $<0.001$ \\
\hline \multicolumn{8}{|c|}{ DMN-SN ( $n=28$, number of observations $=118$ ) } \\
\hline DMN-SN $\times A B \times$ Time & 1.86 & 0.56 & 0.74 to 2.97 & 86 & 3.31 & 0.001 & 0.001 \\
\hline \multicolumn{8}{|c|}{ DMN-FPN ( $n=28$, number of observations $=118$ ) } \\
\hline$D M N-F P N \times A \beta \times$ Time & -0.62 & 0.97 & -2.55 to 1.30 & 86 & -0.64 & 0.52 & 0.52 \\
\hline
\end{tabular}

Regression models are adjusted for age, intracranial volume, sex and education

Beta coefficients are unstandardized

$A \beta$ amyloid-beta, $C l$ confidence interval, $D A N$ dorsal attention network, $D F$ degrees of freedom, $D M N$ default mode network, $F P N$ frontoparietal network, SE standard error, $S N$ salience network

negativity (0.9), the cutoff (1.1), slightly elevated $A \beta$ (1.3), moderately elevated $\mathrm{A} \beta$ (1.5), and high $\mathrm{A} \beta(1.7)$.

Figure 2 shows that the dose-response relationship between $\mathrm{A} \beta$ and memory decline is modulated by both diagnosis and the direction of DMN-DAN correlations. Within the cognitively normal group, negative DMN-DAN correlations have a curvilinear dose-response relationship with memory decline. However, when networks are positively correlated, we found no significant association between $\mathrm{A} \beta$ and memory decline. Conversely, in the MCI group, positive DMN-DAN correlations were associated with a linear dose-response relationship between $A \beta$ and rate of memory. No moderation effects of $A \beta$ were found for the $\mathrm{MCI}$ group with negative correlations, although linear effects approached significance (Table 4).

Power estimations were performed in the cognitively normal group to investigate the added value of adding functional inter-network connectivity to higher levels of $\mathrm{A} \beta$ burden as an inclusion criterion on the required sample size per arm in clinical trials (Fig. 3).

Table 4 Curvilinear associations between functional inter-network connectivity, amyloid-beta burden, and memory decline

\begin{tabular}{|c|c|c|c|c|c|c|c|}
\hline Fitted model & Est. & SE & $95 \% \mathrm{Cl}$ & DF & T value & $p$ value & $p$ value (FDR) \\
\hline \multicolumn{8}{|c|}{ Whole sample, excluding AD group ( $n=91$, number of observations $=364$ ) } \\
\hline \multicolumn{8}{|c|}{ DMN-DAN ( $n=91$, number of observations = 364) } \\
\hline DMN-DAN $\times A \beta \times\left(\right.$ Time $\left.^{2}\right)$ & 0.42 & 0.16 & 0.10 to 0.74 & 266 & 2.60 & 0.01 & 0.02 \\
\hline \multicolumn{8}{|c|}{ DMN-SN ( $n=91$, number of observations $=364)$} \\
\hline DMN-SN $\times$ A $\beta \times\left(\right.$ Time $\left.^{2}\right)$ & 0.25 & 0.14 & -0.02 to 0.52 & 266 & 1.84 & 0.07 & 0.07 \\
\hline \multicolumn{8}{|c|}{ Subset of participants with positive correlation between DMN and DAN ${ }^{b}$} \\
\hline \multicolumn{8}{|c|}{ Whole sample, excluding AD group ( $n=39$, number of observations $=157$ ) } \\
\hline$A \beta \times$ Time & -0.43 & 0.15 & -0.73 to -0.13 & 116 & -2.83 & 0.01 & 0.01 \\
\hline \multicolumn{8}{|c|}{ CN $(n=10$, number of observations $=43)$} \\
\hline$A \beta \times$ Time & 0.22 & 0.20 & -0.19 to 0.63 & 31 & 1.08 & 0.29 & 0.29 \\
\hline \multicolumn{8}{|c|}{$\mathrm{MCl}(n=29$, number of observations $=114)$} \\
\hline$A \beta \times$ Time & -0.44 & 0.13 & -0.69 to -0.18 & 83 & -3.43 & 0.001 & 0.001 \\
\hline \multicolumn{8}{|c|}{ Subset of participants with negative correlation between DMN and DAN ${ }^{\mathrm{a}}$} \\
\hline \multicolumn{8}{|c|}{ Whole sample. Excluding AD group ( $n=52$, number of observations $=207$ ) } \\
\hline$A \beta \times\left(\right.$ Time $\left.^{2}\right)$ & -0.05 & 0.01 & -0.07 to -0.03 & 152 & -4.23 & $<0.001$ & $<0.001$ \\
\hline \multicolumn{8}{|c|}{$\mathrm{CN}(n=18$, number of observations $=75)$} \\
\hline$A \beta \times\left(\right.$ Time $\left.^{2}\right)$ & -0.25 & 0.08 & -0.41 to -0.10 & 53 & -3.33 & 0.001 & 0.001 \\
\hline \multicolumn{8}{|c|}{$\mathrm{MCl}(n=34$, number of observations $=132)$} \\
\hline$A \beta \times$ Time & -0.26 & 0.15 & -0.56 to 0.04 & 96 & -1.73 & 0.09 & 0.09 \\
\hline
\end{tabular}

Regression models are adjusted for age, intracranial volume, sex and education

Beta coefficients are unstandardized

$A \beta$ amyloid-beta, $A D$ Alzheimer's disease, $C l$ confidence interval, $C N$ cognitively normal, $D A N$ dorsal attention network, $D F$ degrees of freedom, $D M N$ default mode network, $F D R$ false discovery rate, $M C I$ mild cognitive impairment, $S E$ standard error, $S N$ salience network

${ }^{\text {a }}$ Models include a quadratic term only if it explained more variance than the linear model without quadratic term 
Networks positively correlated $(n=39)$

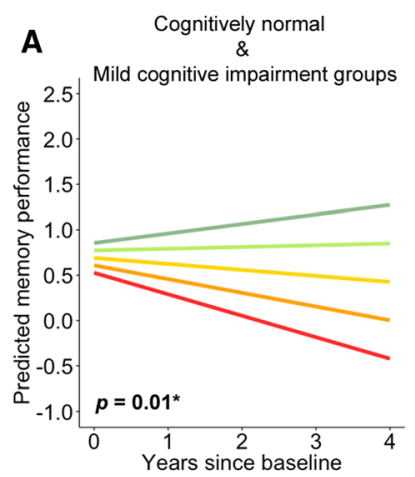

C

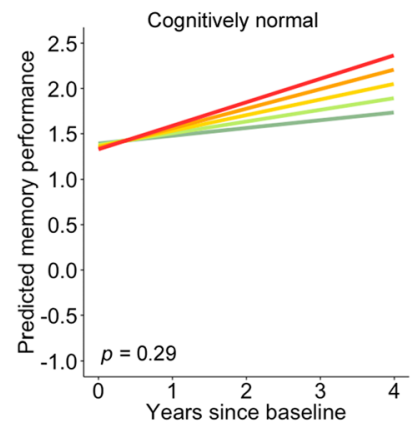

18F-AV-45 Florbetapir standardized uptake value ratio

0.9

$=1.1$

$=1.3$

$=1.5$

\section{D}

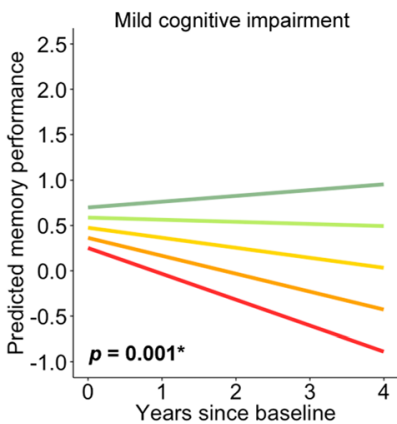

Networks negatively correlated $(n=52)$
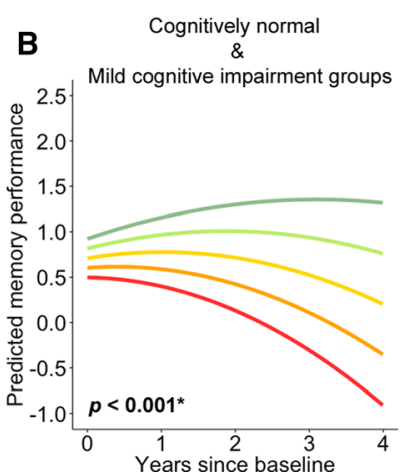

18F-AV-45 Florbetapir

standardized

uptake value ratio

$=0.9$

$=1.1$

$-1.3$

$=1.5$

$=1.7$

Fig. 2 Curvilinear three-way interaction of functional connectivity, amyloid-beta and time and its relationship with memory performance. To visualize the three-way interaction, the whole sample, excluding the AD group, was split based on whether functional connectivity between DMN-DAN was below or above zero, allowing us to visualize the dose-response relationship of A $\beta$ burden (measured with ${ }^{18} \mathrm{~F}-\mathrm{AV}$-45 florbetapir standardized uptake value ratio) on memory decline in both subgroups. ${ }^{18} \mathrm{~F}-\mathrm{AV}-45$ florbetapir standardized uptake value ratio values over 1.11 indicate A $\beta$ positivity. Linear or curvilinear graphs were drawn based on which type of association showed the best fit for each model. All $p$ values are FDR-corrected. Significant effects are indicated by an asterisk and bold font. a Effects within the subgroup of cognitively normal participants and $\mathrm{MCl}$ patients showing positive correlations between networks. $\mathbf{b}$ Curvilinear effects within the subgroup of cognitively normal participants and $\mathrm{MCl}$ patients showing negative correlations between networks. c Effects within the cognitively normal subgroup with positive correlations between networks; no significant dose-response relationship of A $\beta$ on memory (practice effects). $\mathbf{d}$ Effects within the MCI subgroup with positive correlations between networks showing a dose-response relationship of A $\beta$ on memory. e Effects within the cognitively normal subgroup with negative correlations between networks showing a curvilinear dose-response relationship of $A \beta$ on memory. $\mathbf{f}$ Effects within the $\mathrm{MCl}$ subgroup with negative correlations between networks showing no (borderline) significant effect of A $\beta$ burden on memory

These results show that selecting cognitively normal participants with higher levels of $A \beta$ burden and who also have a negative DMN-DAN correlation may greatly reduce the sample size needed to detect amyloid-related changes in memory decline in clinical trials as compared with only including preclinical $\mathrm{AD}$ individuals or those with positive correlations. For instance, the required sample size per arm to detect an effect size of $0.3(30 \%)$ can be reduced by $88 \%$ when using both amyloid and negative inter-network correlations $(n=37)$ as inclusion criteria in clinical trials towards asymptomatic $\mathrm{AD}$, as opposed to considering only amyloid $(n=318)$ in the inclusion process.

While functional inter-network connectivity is a relatively affordable and easy measure to collect in participants, this additional requirement can increase the rate of screen failures at inclusion.

\section{Discussion}

This study provides evidence for a dose-response relationship between $A \beta$ burden and inter-network connectivity on memory decline over 4 years in both healthy and patient populations. Previous studies suggested that negative correlations between task-positive and task-negative networks might have a beneficial effect on memory in young individuals $[13,14]$. We now show that in older adults these associations depend on disease stage and the burden of $A \beta$. In cognitively normal older individuals, negative correlations were associated with memory decline, and this association became stronger in individuals with higher levels of $A \beta$. Conversely, in patients with $\mathrm{MCI}$, the magnitude of $\mathrm{A} \beta$ burden predicted the rate of memory decline associated with positive inter-network correlations. 


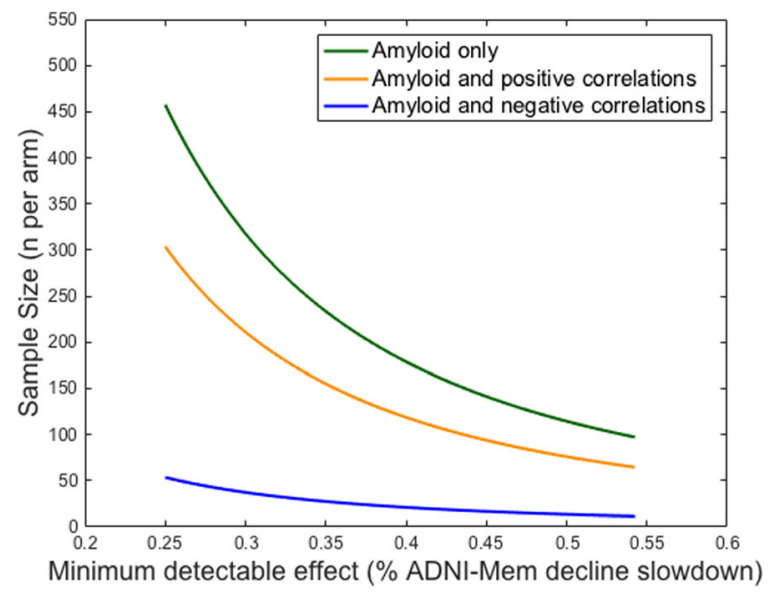

Fig. 3 Power analysis in the cognitively normal group. To assess the effect of including inter-network connectivity as an inclusion criterion in clinical trials, we performed a power analysis in the cognitively normal group. The $x$ axis describes the memory slope reduction in percentages and the $y$ axis describes the number of participants needed per arm to detect this reduction with $80 \%$ power and $a=0.05$ in a 4-year trial with annual assessments. The green line shows the sample size needed when only amyloid is used as an inclusion criterion and inter-network connectivity between the DMN and DAN is not considered. The orange line shows the required sample size when positive inter-network correlations between the DMN and DAN and amyloid are used as inclusion criteria. The blue line shows the required sample size when negative inter-network correlations between the DMN and DAN and amyloid are used as inclusion criteria

These results suggest that the direction of baseline inter-network connectivity can provide additional information when combined with baseline $A \beta$ burden about the rate of expected memory decline and can add important information to the complexity of factors contributing to cognitive decline. Additionally, these findings can have prognostic implications when using functional connectivity as a potential biomarker and show the importance of including both the direction of inter-network connectivity and $\mathrm{A} \beta$ burden for projecting cognitive trajectories as outcome, especially in preclinical AD.

Interestingly, the associations we observed involved mainly the DMN-DAN correlations. The DMN is one of the most investigated networks and is among the first networks where $A \beta$ accumulates significantly early in the disease [41]. It is therefore likely that other functional networks, especially networks tightly coupled to the DMN, are indirectly susceptible to A $\beta$-related alterations. Animal and electrophysiological studies have provided evidence that $\mathrm{A} \beta$ burden leads to synaptic dysfunction and network disorganization [42].

Widespread inter-network reorganization under the influence of $A \beta$ have indeed been reported for the coupling between DMN and DAN [43], DMN and SN [19], and DMN and FPN [43], suggesting that topographical closeness to certain hubs, such as the association cortices of the
$\mathrm{DMN}$, may put networks at increased risk for $\mathrm{A} \beta$ toxicity. Our findings now show that functional implications of the interactions between large-scale brain networks and amyloid in relation to memory decline were mainly observed for the DMN-DAN correlations, with borderline significant associations for the DMN-SN correlations. This is in line with previous research suggesting that an optimal interaction between these specific networks is important for cognitive functioning $[13,14,44]$. The fact that we observed associations between greater positive inter-network correlations and better memory performance over time in clinically normal individuals suggests the presence of compensation mechanisms involving possible reconfiguration of large-scale networks $[45,46]$. In the context of preclinical $\mathrm{AD}$, this compensation may provide the architectural basis for maintaining optimal memory performance in old age [45, 47]. These compensation mechanisms may be moderated by cognitive reserve [46]. Individuals with preclinical $\mathrm{AD}$ may be able to compensate for $\mathrm{AD}$ pathology in the form of positive inter-network correlations. Furthermore, previous studies reported differential connectivity patterns for the anterior versus posterior DMN [48]. Increased within-network functional connectivity in the anterior DMN was associated with higher levels of amyloid pathology in preclinical AD [21], indicative of regional compensatory mechanisms. However, as the disease progresses, compensatory mechanisms may be dependent on cognitive reserve to stave off memory decline. Attenuated negative correlations between the DMN-DAN may signal impending neuronal breakdown resulting from $A \beta$ toxicity in the prodromal phase, a finding consistent with previous work [46].

The DMN and DAN may operate in a negative correlation pattern to facilitate attention-related processes during cognitive tasks. The DAN is thought to coordinate cross-talk between brain networks, especially during memory tasks. The coordination between brain networks may become especially important in MCI, where medial temporal lobe structures become functionally isolated from other regions or networks. In $\mathrm{MCI}$, a coordinated antiphase functional connection between the DMN and DAN may be fundamental in maintaining optimal levels of memory performance [46].

Finally, functional connectivity deteriorates as the disease progresses to more advanced stages [48]. This may partially explain the lack of connectivity-related findings in the $\mathrm{AD}$ group, as coherent connectivity patterns between networks may dissipate leaving no association between inter-network connectivity and cognition.

Our dose-response findings relating $A \beta$ burden to memory decline, depending on functional connectivity status, showed different patterns between cognitively normal individuals and patients with MCI. This has important implications for early detection of individuals at risk of 
cognitive decline and the selection of preclinical and prodromal $\mathrm{AD}$ individuals for trials using a combination of functional connectivity and $A \beta$. Patients with MCI showed a dose-response relationship between $A \beta$ and memory decline for positive DMN-DAN correlations. In clinically normal individuals, $A \beta$ has a dose-response relationship with memory decline when DMN-DAN correlations are negative. Selecting preclinical AD individuals with positive DMN-DAN correlations could lead to the selection of individuals where individuals with higher levels of $A \beta$ have similar practice effects as individuals with lower levels of $A \beta$ burden. Thus, the direction of inter-network connectivity in combination with $A \beta$ burden can have important implications for participant selection in clinical trials. This notion is further outlined by the results from our power analysis showing that the selection of cognitively normal individuals with elevated $A \beta$ levels and negative inter-network correlations reduced the required sample size by $88 \%$ to slow down memory decline by $30 \%$.

Furthermore, we did not observe independent or synergistic effects of inter-network connectivity or $A \beta$ on cognition in patients with $\mathrm{AD}$ in the 2-year follow-up period. This could be related to widespread deposits of both $A \beta$ and tau that may have impacted the integrity of the entire brain. We also did not observe between-group differences in inter-network connectivity of the DMN-DAN at baseline, which is in contrast to previous reports in similar populations [17-19]. These discrepancies may be related to sample size, methodological differences (regional versus network-based analyses), and the larger age range of the participants in our study.

Interestingly, the results found in our study seem to be domain-specific for memory. This may be explained by the DMN being specifically associated with memory functions $[10,46]$. Since we found the most convincing results in the DMN-DAN correlation, this may emphasize the domain-specific nature of the interaction between DMN-DAN correlation, amyloid burden, and memory decline in preclinical AD.

\section{Limitations}

Due to the observational nature of our study, our data do not allow for any causal inferences between changes in functional connectivity between networks or $\mathrm{A} \beta$ deposition. Determining the temporal direction of the three-way association between amyloid, memory decline, and inter-network functional connectivity requires further experimental investigation. Furthermore, longitudinal data of the $\mathrm{AD}$ group are limited to a follow-up period of 2 years. This may partially explain the lack of significant results found in this group, although it is also possible that the disease process and associated pathological accumulations are too widespread and may have affected functional networks in multiple ways, all associated with cognitive decline.

\section{Future directions}

With the recent development of tau positron emission tomography tracers, future studies can investigate how tau pathology may impact inter-network connectivity since research has suggested a dynamic influence of tau on connectivity within networks, depending on $A \beta$ levels [49-51]. Results from such a study could ultimately culminate in clinical trials where participants can be chosen using multi-modal selective criteria for memory decline due to $\mathrm{AD}$.

Additionally, future studies should investigate whether carriers of the apolipoprotein $\varepsilon 4$ allele show stonger synergistic effects between $\mathrm{AD}$ pathology $[52,53]$ and inter-network correlations on cognitive decline. Such investigations may further refine the selection criteria for trials and may have implications for determining response to treatment.

Finally, previous research has shown that $\mathrm{AD}$ variants, such as posterior cortical atrophy and early-onset $\mathrm{AD}$, may have unique networks that are preferentially affected in the disease process [54]. Thus, future research may want to investigate how $A \beta$, inter-network correlations, and cognitive decline are associated in these different variants of $\mathrm{AD}$ and whether inter-network correlations may be associated with different cognitive domains in these variants.

\section{Conclusions}

In conclusion, our results show that the direction of inter-network connectivity provides additional information to baseline $A \beta$ burden about the rate of expected memory decline. These findings add important information for the understanding of factors contributing to cognitive decline. These results also suggest that when using functional connectivity as a biomarker or selection criterion for trials in preclinical populations, the directionality of inter-network connectivity might aid in selecting individuals that are more likely to be on an $A \beta$-related negative memory trajectory. Including information about both $A \beta$ burden and inter-network functional connectivity can improve recruitment strategies and decrease the time to determine the efficacy of clinical trials in the asymptomatic phase of the disease.

\section{Additional file}

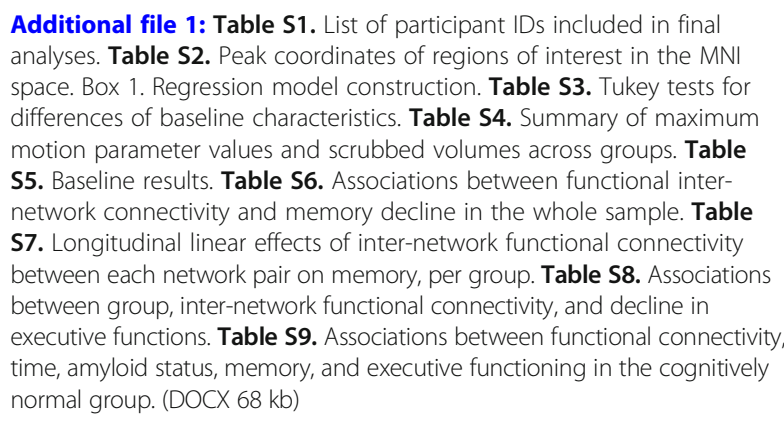




\section{Abbreviations}

A $\beta$ : Amyloid-beta; AD: Alzheimer's disease; ADNI: Alzheimer's Disease Neuroimaging Initiative; DAN: Dorsal attention network; DMN: Default mode network; FDR: False-discovery rate; FPN: Frontoparietal network; MCl: Mild cognitive impairment; ROI: Region of interest; SN: Salience network

\section{Acknowledgements}

We would like to thank Ed H.B.M. Gronenschild, PhD (Faculty of Health, Medicine and Life Sciences, School for Mental Health and Neuroscience, Department of Psychiatry and Neuropsychology, Alzheimer Center Limburg, Maastricht University, Maastricht, The Netherlands) for his invaluable assistance in the processing of imaging data.

Data used in the preparation of this article were obtained from the Alzheimer's Disease Neuroimaging Initiative (ADNI) database (adni.loni.usc.edu). As such, the investigators within the ADNI contributed to the design and implementation of ADNI and/or provided data but did not participate in the analysis or writing of this report. A complete listing of ADNI investigators can be found at https:// adni.loni.usc.edu/wp-content/uploads/how_to_apply/

ADNI_Acknowledgement_List.pdf.

\section{Funding}

HIL is supported by funding from NWO (Veni-451-14-035), the European Union's Horizon 2020 Research and Innovation Programme under the Marie Sklodowska-Curie Grant agreement (IF-2015-GF, 706714), a standard grant of the International Stichting voor Alzheimer Onderzoek (\#15007) and a research grant by the Deutsche Forschungsgemeinschaft (JA/2336/1-1). ADNI is funded through generous contributions from the following: AbbVie, Alzheimer's Association; Alzheimer's Drug Discovery Foundation; Araclon Biotech; BioClinica, Inc.; Biogen; Bristol-Myers Squibb Company; CereSpir, Inc.; Cogstate; Eisai Inc.; Elan Pharmaceuticals, Inc.; Eli Lilly and Company; Eurolmmun; F. Hoffmann-La Roche Ltd. and its affiliated company Genentech, Inc.; Fujirebio; GE Healthcare; IXICO Ltd.; Janssen Alzheimer Immunotherapy Research \& Development, LLC.; Johnson \& Johnson Pharmaceutical Research \& Development LLC.; Lumosity; Lundbeck; Merck \& Co., Inc.; Meso Scale Diagnostics, LLC.; NeuroRx Research; Neurotrack Technologies; Novartis Pharmaceuticals Corporation; Pfizer Inc. Piramal Imaging; Servier; Takeda Pharmaceutical Company; and Transition Therapeutics. The Canadian Institutes of Health Research is providing funds to support ADNI clinical sites in Canada. Private sector contributions are facilitated by the Foundation for the National Institutes of Health (www.fnih.org). The grantee organization is the Northern California Institute for Research and Education, and the study is coordinated by the Alzheimer's Therapeutic Research Institute at the University of Southern California. ADNI data are disseminated by the Laboratory for Neuro Imaging at the University of Southern California and funded by the National Institute on Aging, the National Institute of Biomedical Imaging and Bioengineering. Data collection and sharing for this project was funded by the Alzheimer's Disease Neuroimaging Initiative (ADNI) (National Institutes of Health Grant U01 AG024904) and DOD ADNI (Department of Defense award number W81XWH-12-2-0012). The funding sources had no involvement related to this manuscript in any way.

\section{Availability of data and materials}

The datasets generated and/or analyzed during the current study are available in the ADNI repository, http://adni.loni.usc.edu/data-samples/access-data/.

\section{Authors' contributions}

All authors contributed to the conception and design of the study. RWEVH and HILJ performed analyses on the data. All authors interpreted the data. RWEVH drafted the initial version of the manuscript. JMR and HILJ revised the manuscript for important intellectual content, and all authors read and approved the final manuscript.

\section{Ethics approval and consent to participate}

ADNI is a multisite investigation. All ADNI sites obtained Institutional Review Board approval for the use of humans for research from their respective Institutional Review Board within 5 months of receiving the final protocol and all participants provided written informed consent.

\section{Consent for publication}

Not applicable.

\section{Competing interests}

The authors declare that they have no competing interests.

\section{Publisher's Note}

Springer Nature remains neutral with regard to jurisdictional claims in published maps and institutional affiliations.

\section{Author details}

${ }^{1}$ Faculty of Health, Medicine and Life Sciences; School for Mental Health and Neuroscience, Department of Psychiatry and Neuropsychology, Alzheimer Center Limburg, Maastricht University, Dr. Tanslaan 12, 6229 ET Maastricht, the Netherlands. ${ }^{2}$ Department of Anesthesiology, Sankt-Willibrord Spital, Emmerich, Germany. ${ }^{3}$ Faculty of Psychology and Neuroscience, Department of Cognitive Neuroscience, Maastricht University, Maastricht, The Netherlands. ${ }^{4}$ Division of Nuclear Medicine and Molecular Imaging, Department of Radiology, Massachusetts General Hospital/Harvard Medical School, Boston, MA, USA.

Received: 9 May 2018 Accepted: 7 August 2018

Published online: 28 August 2018

\section{References}

1. McKhann G, Knopman DS, Chertkow H, Hyman BT, Jack CR Jr, Kawas $\mathrm{CH}$, et al. The diagnosis of dementia due to Alzheimer's disease: recommendations from the National Institute on Aging-Alzheimer's Association workgroups on diagnostic guidelines for Alzheimer's disease. Alzheimers Dement. 2011;7:263-9.

2. Jagust WJ, Mormino EC. Lifespan brain activity, beta-amyloid, and Alzheimer's disease. Trends Cogn Sci. 2011;15:520-6.

3. Jack CR, Knopman DS, Jagust WJ, Petersen RC, Weiner MW, Aisen PS, et al Tracking pathophysiological processes in Alzheimer's disease: an updated hypothetical model of dynamic biomarkers. Lancet Neurol. 2013;12:207-16.

4. Price $J$, Davis $P$, Morris J, White D. The distribution of tangles, plaques and related immunohistochemical markers in healthy aging and Alzheimer's disease. Neurobiol Aging. 1991;12:295-312.

5. Mulder C, Verwey NA, van der Flier WM, Bouwman FH, Kok A, van Elk EJ, et al. Amyloid- $\beta$ (1-42), total tau, and phosphorylated tau as cerebrospinal fluid biomarkers for the diagnosis of Alzheimer disease. Clin Chem. 2010;56:248-53.

6. Braak H, Braak E. Neuropathological staging of Alzheimer-related changes. Acta Neuropathol. 1991;82:239-59.

7. Farrell ME, Kennedy KM, Rodrigue KM, et al. Association of longitudinal cognitive decline with amyloid burden in middle-aged and older adults: evidence for a dose-response relationship. JAMA Neurol. 2017:74:830-8.

8. Boyle PA, Wilson RS, Yu L, Barr AM, Honer WG, Schneider JA, et al. Much of late life cognitive decline is not due to common neurodegenerative pathologies. Ann Neurol. 2013;74:478-89.

9. Gauthier S, Albert M, Fox N, Goedert M, Kivipelto M, Mestre-Ferrandiz J, et al. Why has therapy development for dementia failed in the last two decades? Alzheimers Dement. 2016;12:60-4

10. Damoiseaux JS, Beckmann CF, Arigita EJS, Barkhof F, Scheltens P, Stam CJ, et al. Reduced resting-state brain activity in the "default network" in normal aging. Cereb Cortex. 2008;18:1856-64.

11. Andrews-Hanna JR, Snyder AZ, Vincent JL, Lustig C, Head D, Raichle

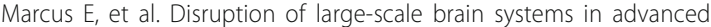
aging. Neuron. 2007;56:924-35.

12. Buckley RF, Schultz AP, Hedden T, Papp KV, Hanseeuw BJ, Marshall G, et al. Functional network integrity presages cognitive decline in preclinical Alzheimer disease. Neurology. 2017;89(1):29-37.

13. Fornito A, Harrison BJ, Zalesky A, Simons JS. Competitive and cooperative dynamics of large-scale brain functional networks supporting recollection. Proc Natl Acad Sci U S A. 2012;109:12788-93.

14. Kelly AM, Uddin LQ, Biswal BB, Castellanos FX, Milham MP. Competition between functional brain networks mediates behavioral variability. Neurolmage. 2008;39:527-37.

15. Fox MD, Snyder AZ, Vincent JL, Corbetta M, Van Essen DC, Raichle ME. The human brain is intrinsically organized into dynamic, anticorrelated functional networks. Proc Natl Acad Sci U S A. 2005:102:9673-8.

16. Pinsk MA, Kastner S. Unconscious networking. Nature. 2007;447:46. 
17. Wang K, Liang M, Wang L, Tian L, Zhang X, Li K, et al. Altered functional connectivity in early Alzheimer's disease: a resting-state fMRI study. Hum Brain Mapp. 2007;28:967-78.

18. Esposito R, Cieri F, Chiacchiaretta P, Cera N, Lauriola M, Di Giannantonio M, et al. Modifications in resting state functional anticorrelation between default mode network and dorsal attention network: comparison among young adults, healthy elders and mild cognitive impairment patients. Brain Imaging Behav. 2018;12:127-41.

19. Brier MR, Thomas JB, Snyder AZ, Benzinger TL, Zhang D, Raichle ME, et al. Loss of intranetwork and internetwork resting state functional connections with Alzheimer's disease progression. J Neurosci. 2012;32:8890-9.

20. Mormino EC, Betensky RA, Hedden T, Schultz AP, Amariglio RE, Rentz DM, et al. Synergistic effect of beta-amyloid and neurodegeneration on cognitive decline in clinically normal individuals. JAMA Neurol. 2014;71:1379-85.

21. Mormino EC, Smiljic A, Hayenga AO, Onami SH, Greicius MD, Rabinovici $\mathrm{GD}$, et al. Relationships between beta-amyloid and functional connectivity in different components of the default mode network in aging. Cereb Cortex. 2011;21:2399-407.

22. Sheline YI, Raichle ME, Snyder AZ, Morris JC, Head D, Wang S, et al. Amyloid plaques disrupt resting state default mode network connectivity in cognitively normal elderly. Biol Psychiatry. 2010:67:584-7.

23. Mueller SG, Weiner MW, Thal LJ, Petersen RC, Jack C, Jagust W, et al. The Alzheimer's disease neuroimaging initiative. Neuroimaging Clin $\mathrm{N}$ Am. 2005; 15:869-77.

24. Landau SM, Mintun MA, Joshi AD, Koeppe RA, Petersen RC, Aisen PS, et al. Amyloid deposition, hypometabolism, and longitudinal cognitive decline. Ann Neurol. 2012;72:578-86.

25. Petersen RC. Mild cognitive impairment as a diagnostic entity. J Intern Med. 2004; 256:183-94.

26. Petersen RC, Aisen P, Beckett LA, Donohue M, Gamst A, Harvey DJ, et al. Alzheimer's disease neuroimaging initiative (ADNI) clinical characterization. Neurology. 2010;74:201-9.

27. McKhann G, Drachman D, Folstein M, Katzman R, Price D, Stadlan EM. Clinical diagnosis of Alzheimer's disease Report of the NINCDS-ADRDA Work Group under the auspices of Department of Health and Human Services Task Force on Alzheimer's Disease. Neurology. 1984;34:939.

28. Crane PK, Carle A, Gibbons LE, Insel P, Mackin RS, Gross A, et al. Development and assessment of a composite score for memory in the Alzheimer's Disease Neuroimaging Initiative (ADNI). Brain Imaging Behav. 2012;6:502-16

29. Gibbons LE, Carle AC, Mackin RS, Harvey D, Mukherjee S, Insel P, et al. A composite score for executive functioning, validated in Alzheimer's Disease Neuroimaging Initiative (ADNI) participants with baseline mild cognitive impairment. Brain Imaging Behav. 2012;6:517-27.

30. Whitfield-Gabrieli S, Nieto-Castanon A. Conn: a functional connectivity toolbox for correlated and anticorrelated brain networks. Brain connectivity. 2012;2:125-41.

31. Murphy K, Birn RM, Handwerker DA, Jones TB, Bandettini PA. The impact of global signal regression on resting state correlations: are anti-correlated networks introduced? Neurolmage. 2009;44:893-905.

32. Behzadi Y, Restom K, Liau J, Liu TT. A component-based noise correction method (CompCor) for BOLD and perfusion-based fMRI. Neurolmage. 2007;37:90-101.

33. Field A. Discovering statistics using IBM SPSS statistics. London: SAGE Publications Ltd; 2013.

34. Ibrahim JG, Molenberghs G. Missing data methods in longitudinal studies: a review. Test (Madr). 2009:18:1-43.

35. Pinheiro J, Bates D, DebRoy S, Sarkar D, R Core Team. nlme: linear and nonlinear mixed effects models. R package version 3.1-128. 2016. http:// CRAN.R-project.org/package=nlme.

36. Bischof GN, Rodrigue KM, Kennedy KM, Devous MD, Park DC. Amyloid deposition in younger adults is linked to episodic memory performance. Neurology. 2016;87:2562-6.

37. Whitwell JL, Crum WR, Watt HC, Fox NC. Normalization of cerebral volumes by use of intracranial volume: implications for longitudinal quantitative MR imaging. Am J Neuroradiol. 2001;22:1483-9.

38. Fischl B. FreeSurfer. Neuroimage. 2012;62:774-81.

39. Buckner RL, Head D, Parker J, Fotenos AF, Marcus D, Morris JC, et al. A unified approach for morphometric and functional data analysis in young, old, and demented adults using automated atlas-based head size normalization: reliability and validation against manual measurement of total intracranial volume. Neurolmage. 2004:23:724-38.

40. Hanseeuw BJ, Betensky RA, Mormino EC, Schultz AP, Sepulcre J, Becker JA, et al. PET staging of amyloidosis using striatum. Alzheimers Dement. 2018. https://doi.org/10.1016/j.jalz.2018.04.011.

41. Elman JA, Madison CM, Baker SL, Vogel JW, Marks SM, Crowley S, et al. Effects of beta-amyloid on resting state functional connectivity within and between networks reflect known patterns of regional vulnerability. Cereb Cortex. 2016:26:695-707.

42. Mucke L, Selkoe DJ. Neurotoxicity of amyloid $\beta$-protein: synaptic and network dysfunction. Cold Spring Harb Perspect Med. 2012;2:a006338.

43. Grothe MJ, Teipel SJ. Spatial patterns of atrophy, hypometabolism, and amyloid deposition in Alzheimer's disease correspond to dissociable functional brain networks. Hum Brain Mapp. 2016;37:35-53.

44. Keller JB, Hedden T, Thompson TW, Anteraper SA, Gabrieli JD, WhitfieldGabrieli S. Resting-state anticorrelations between medial and lateral prefrontal cortex: association with working memory, aging, and individual differences. Cortex. 2015;64:271-80.

45. Cabeza R, Anderson ND, Locantore JK, Mclntosh AR. Aging gracefully: compensatory brain activity in high-performing older adults. Neurolmage. 2002;17:1394-402.

46. Franzmeier N, Buerger $K$, Teipel S, Stern Y, Dichgans M, Ewers M, et al, Cognitive reserve moderates the association between functional network anti-correlations and memory in MCl. Neurobiol Aging. 2017;50:152-62.

47. Spreng RN, Stevens WD, Viviano JD, Schacter DL. Attenuated anticorrelation between the default and dorsal attention networks with aging: evidence from task and rest. Neurobiol Aging. 2016;45:149-60.

48. Damoiseaux JS, Prater KE, Miller BL, Greicius MD. Functional connectivity tracks clinical deterioration in Alzheimer's disease. Neurobiol Aging. 2012;33:828. e19-.e30

49. Schultz AP, Chhatwal JP, Hedden T, Mormino EC, Hanseeuw BJ, Sepulcre J et al. Phases of hyperconnectivity and hypoconnectivity in the default mode and salience networks track with amyloid and tau in clinically normal individuals. J Neurosci. 2017;37:4323-31.

50. Sepulcre J, Sabuncu MR, Li Q, El Fakhri G, Sperling R, Johnson KA. Tau and amyloid $\beta$ proteins distinctively associate to functional network changes in the aging brain. Alzheimers Dement. 2017:13:1261-9.

51. Jacobs HI, Hedden T, Schultz AP, Sepulcre J, Perea RD, Amariglio RE, et al. Structural tract alterations predict downstream tau accumulation in amyloid-positive older individuals. Nat Neurosci. 2018;21:424.

52. Corder EH, Saunders AM, Strittmatter WJ, Schmechel DE, Gaskell PC, Small $\mathrm{G}$, et al. Gene dose of apolipoprotein E type 4 allele and the risk of Alzheimer's disease in late onset families. Science. 1993;261:921-3.

53. Strittmatter WJ, Saunders AM, Schmechel D, Pericak-Vance M, Enghild J, Salvesen GS, et al. Apolipoprotein E: high-avidity binding to beta-amyloid and increased frequency of type 4 allele in late-onset familial Alzheimer disease. Proc Natl Acad Sci U S A. 1993;90:1977-81.

54. Lehmann M, Madison CM, Ghosh PM, Seeley WW, Mormino E, Greicius MD, et al. Intrinsic connectivity networks in healthy subjects explain clinical variability in Alzheimer's disease. Proc Natl Acad Sci U S A. 2013;110:11606-11.

Ready to submit your research? Choose BMC and benefit from:

- fast, convenient online submission

- thorough peer review by experienced researchers in your field

- rapid publication on acceptance

- support for research data, including large and complex data types

- gold Open Access which fosters wider collaboration and increased citations

- maximum visibility for your research: over $100 \mathrm{M}$ website views per year

At BMC, research is always in progress.

Learn more biomedcentral.com/submission 